\title{
Towards an open 3D participatory citizen debate
}

This paper presents a platform aiming the ease of the debate between citizens. In the early 2010'ies, governments are seeking new ways to be more accountable and transparent towards their citizens; marking a renewal in public participation. In return, citizens are eager to be heard and to use new tools based on information and communication technologies (ICT) like the web 2.0. This public's empowerment presents some costs for the authorities who are mainly concerned with the loss of decision making power. To face those challenges, several 2D online maps have been developed to help the governments to direct and centralize citizens insights. Those previous collaborating mapping tools helped to identify the characteristics of a reliable platform : user-friendly, simple and accessible (anywhere at any time). In our implementation, we adopted the third dimension which provides numerous benefits : 1 . a more effective and effortless visualization, 2. An unbiased representation of the environment, 3. The merge of the participant cognition spaces. From our past experiences, we conceptualized the actors (citizens / facilitator / transcriber) interactions and dynamics in public engagement on-site meeting. From this approach, we evaluated how the utilization of a 3D virtual environment as the support of the participation will reshape and enhance the relation synergies between the actors : 1 . Centralization of the interactions within the platform, 2. Automated analysis from the gathered raw information, 3. Reachability of a larger part of the population, 4. Lightening of the participatory processes. 


\title{
Towards an open 3D participatory citizen debate
}

\author{
Thibaud Chassin ${ }^{1 *}$, Jens Ingensand ${ }^{1}$, Florent Joerin ${ }^{12}$ \\ ${ }^{1}$ INSIT, University of Applied Sciences Western Switzerland, School of Business and \\ Engineering Vaud, Insit, Yverdon-les-Bains Switzerland \\ firstname.lastname@heig-vd.ch \\ ${ }^{2}$ Lausanne University, Lausanne, Switzerland \\ firstname.lastname@unil.ch \\ * Corresponding Author \\ \{thibaud.chassin, jens.ingensand\}@heig-vd.ch
}

\section{ABSTRACT}

This paper presents a platform aiming the ease of the debate between citizens. In the early 2010'ies, governments are seeking new ways to be more accountable and transparent towards their citizens; marking a renewal in public participation. In return, citizens are eager to be heard and to use new tools based on information and communication technologies (ICT) like the web 2.0. This public's empowerment presents some costs for the authorities who are mainly concerned with the loss of decision making power. To face those challenges, several 2D online maps have been developed to help the governments to direct and centralize citizens insights. Those previous collaborating mapping tools helped to identify the characteristics of a reliable platform: user-friendly, simple and accessible (anywhere at any time). In our implementation, we adopted the third dimension which provides numerous benefits: 1 . a more effective and effortless visualization, 2. An unbiased representation of the environment, 3. The merge of the participant cognition spaces. From our past experiences, we conceptualized the actors (citizens / facilitator / transcriber) interactions and dynamics in public engagement on-site meeting. From this approach, we evaluated how the utilization of a $3 \mathrm{D}$ virtual environment as the support of the participation will reshape and enhance the relation synergies between the actors: 1. Centralization of the interactions within the platform, 2. Automated analysis from the gathered raw information, 3. Reachability of a larger part of the population, 4 . Lightening of the participatory processes.

Keywords: Open debate, 3D virtual environment, Open-Government, Public engagement. 


\section{INTRODUCTION}

With the introduction of web 2.0 technologies, the concept of e-Government has been developed. This concept puts citizens at the heart of governmental policies and allows them to, for instance, consult documents online and to declare their taxes. During the 2010'ies the same core values were used to introduce the idea of open-Government. This idea goes further, aiming at delivering open data and processes to the public. Several initiatives can be found on governmental / cities websites; e.g. in Switzerland (egovernment.ch), in the UK

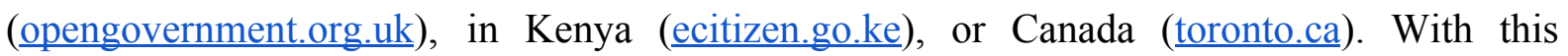
approach of openness and the use of web 2.0 tools, the authorities have the opportunity to enhance their transparency, accountability and efficiency.

In his speech, former president Obama, acknowledged the needs of involving citizens in public decisions.

"Our commitment to openness means more than simply informing the American people about how decisions are made. It means recognizing that government does not have all the answers, and that public officials need to draw on what citizens know." (Obama, 2009)

This speech led to the establishment of the first executive order aiming at creating the Open-Government Initiative. The keywords linked to this initiative are : accountability, transparency, participation and collaboration.

However, these benefits come with costs and risks such as the loss of control towards citizens, the experiences of critical reviews or issues with data privacy and security outbreaks (Sivarajah, Irani \& al., 2015).

\section{A PLATFORM TO SUPPORT CITIZENS OPEN DEBATE}

Up to now several 2D mapping platforms have been developed to fulfill the demand of engaging citizens. Some key points can be highlighted from these use cases. (Bugs, Granell \& al., 2010) for instance have created a collaborative mapping tool with a user friendly interface and easy to use features with low entry cost (no software installation nor background knowledge required). This studies demonstrates that users have acknowledged the usefulness of the tool and are eager to participate more often with this kind of online platforms. In their project, (Fechner \& Kray, 2014) designed a "Dialog Map" where open issues can be discussed. The authors point out the value of offering easily accessible data and well-designed content visibility. 
Today only very few open Government projects focus on 3D visualization. One example is the Cityplanner project (ityplanneronline.com), which provides features such as the possibility to visualize $3 \mathrm{D}$ buildings and to communicate with the citizens regarding future projects.

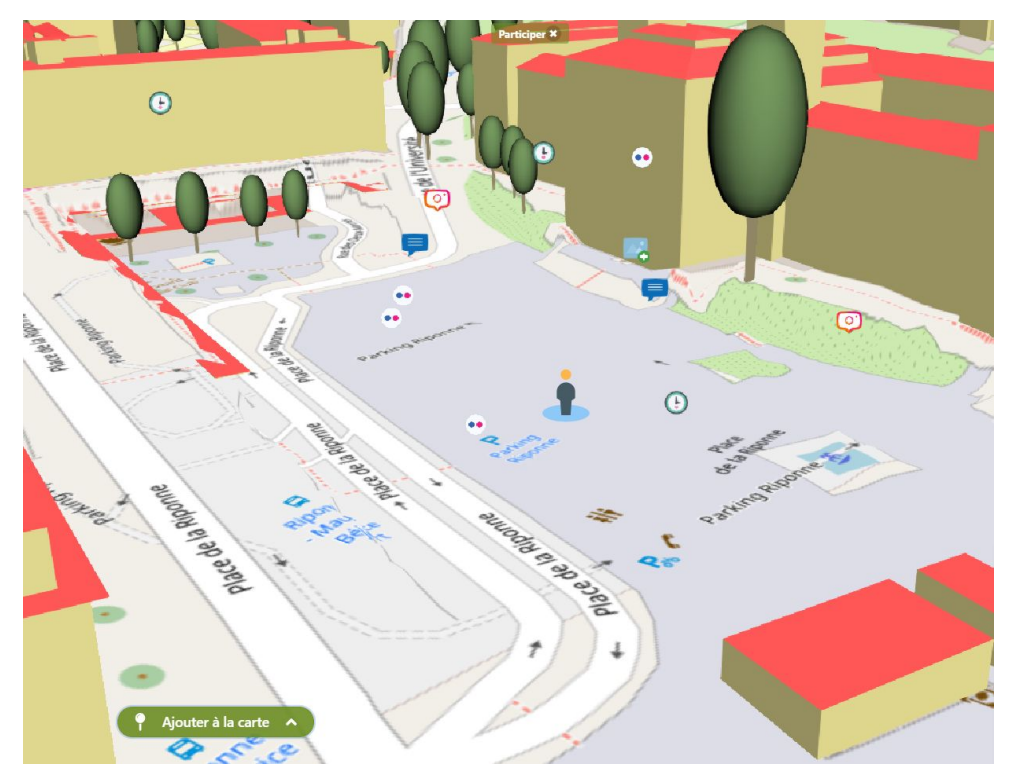

Figure 1: The 3D platform as the core of interactions between citizens. On this screenshot we can see varying content displayed as icons : comments, uploaded pictures, screenshots, historic photographs and flickR posts.

In this paper, we introduce a 3D virtual platform to facilitate an open debate between citizens and stakeholders about urban planning using 3D data. Georeferenced information such as mockups of planned buildings, historical pictures as well as content coming from social networks (e.g. filtered flickR (flickr.com) images) and open questions are elements provided to the users. In this platform a citizen can add a pin to the virtual environment and attach a comment, a picture, a screenshot as well as sketches. Moreover a citizen can answer questions of the stakeholders and comment on other citizens' contributions. All information added by citizens follows a validation workflow allowing the platform to keep an atmosphere of respect between participants. The platform (figure 1) has been designed to serve as the core of an open debate in a participatory process. Our platform centralizes all exchange fluxes between participants and facilitators (community managers, city representatives, urban designers, experts) within the 3D virtual scene. The interface is based on open-sources libraries such as CesiumJS (cesiumjs.org) and vue.js (vuejs.org). All interactions with the platform are stored in an open-source georeferenced database, PostgreSQL (postgresql.org). Our interest in using 3D data is to provide a more effective and effortless representation of the environment as compared to $2 \mathrm{D}$ maps. Another advantage is transparency - it is for instance impossible to use the bias of perspective (e.g. illustrations taken from a certain point 
of view) in order to argument for or against a project due to the fact that all users can visualize the same scene from different angles and perspectives.

Each citizen creates a unique mental model of the environment. The possibility to use a 3D platform allows the user to utilize a less abstract representation of the environment that is easier to match with his own mental model. Therefore the utilization of a common platform enables merging the mental models of all participants into a common and neutral cognition space that allows for objective discussions.

\section{THE ROLE OF THE 3D PLATFORM IN CITIZENS ENGAGEMENT PROCESSES}

Three concepts can be defined in the context of citizen engagement processes where citizen actively interact with each other: "Citizens", "Facilitators" and "Transcribers". The "Citizens"-concept represents the public affected or concerned by a specific urban project. The "Facilitators" moderate, guide, or answer a citizen's concerns. The "Transcribers" (e.g. scribe, automated scripts) symbolize the post-processing of the collected data aiming at reporting clear results of the debate. Figure 2 presents the interactions of an usual public participation meeting based on our observations and experiences into the organisation of public events (on the left). On the right, those same actor interactions reshaped by the utilization of our platform.

During an onsite public session citizen interact both with each other and the facilitator. Discussions can be stimulated using external content (either provided by facilitators or other citizens) such as 2D maps, pictures, text. A mindful transcriber has to gather all information exchanges to produce a report highlighting the main topics that have been addressed. In each session a small sample of the affected population (50-150 citizen) is selected and divided into small working groups (usually 10 participants). With the assistance of one (or two) facilitator(s) and one transcriber, the citizens are encouraged to address a specialized topic (e.g. infrastructure, mobility, environment, etc.).

Traditional public meetings however have some limits: onsite meetings require actors to be present at the same place at the same time. This can imply difficulties in reaching some parts of the population. Besides, only a limited number of citizen can participate at the same time. Moreover, several discussions can happen at the same time and it can be a challenge for the transcriber to collect all information. Finally, addressing delicate issues in terms of urban planning can lead to emotional reactions which can influence the transcriber's report. (Harvey, 2009) 
In our approach we use a 3D platform at the core of the debate. Citizens interact with other citizens and Facilitators within the 3D scene. Their contributions involve debate participation, such as asking questions or adding insights. Simultaneously an automated script collects all data and produces an automated report of the current state of the discussion. Several benefits results from this concept such as an asynchronous debate and a 24/7 service that allows citizens to participate independently from time and space. Thereby a larger part of the population can be reached (improvement of the accessibility).
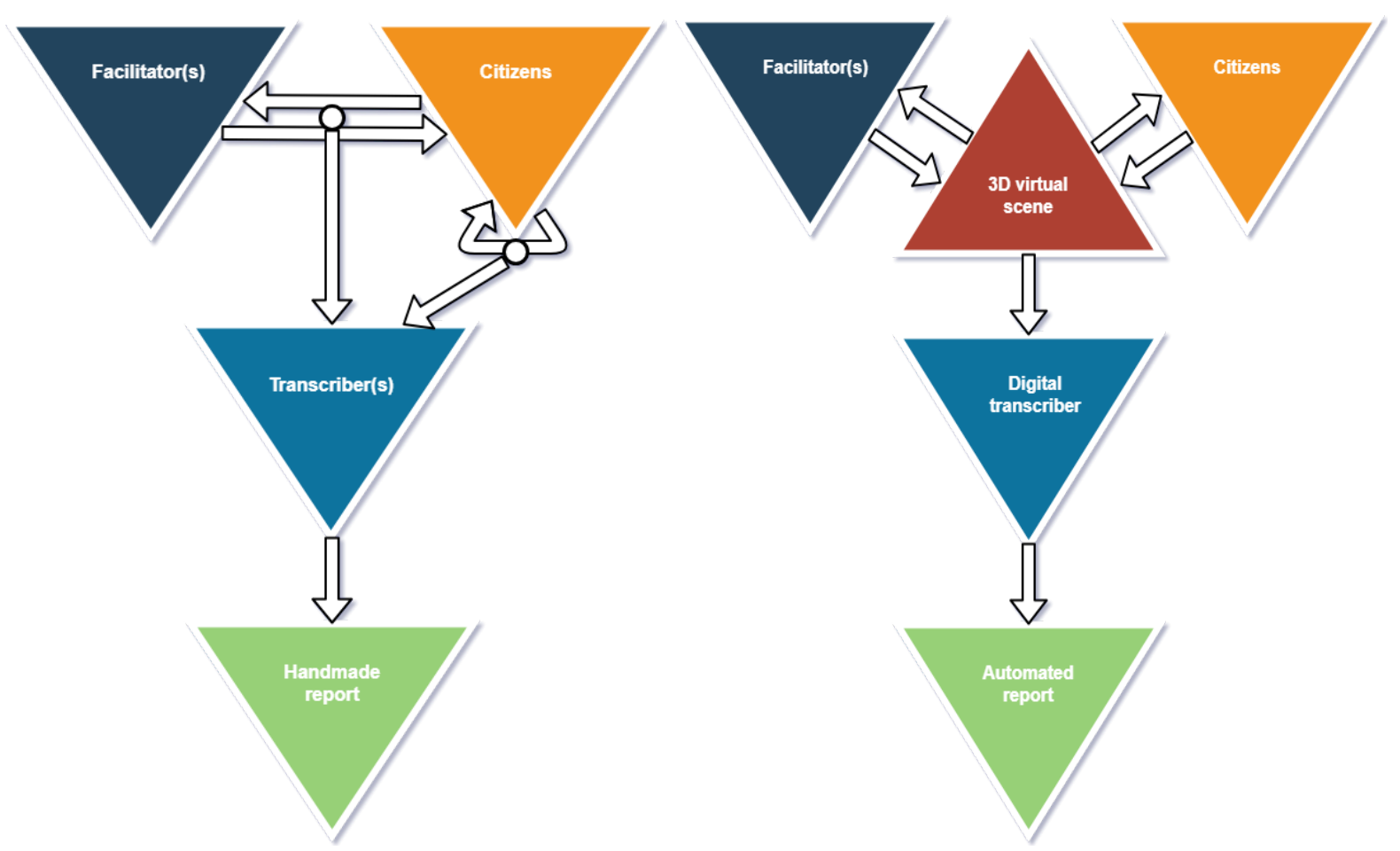

Figure 2: Traditional public engagement meetings vs the use of a 3D platform. On the left, a typical scheme is shown, where Citizens either exchange directly or with a Facilitator ; all information is caught by a Transcriber. On the right, the $3 \mathrm{D}$ platform within a public engagement process. All discussions are centralized in one scene and automatically captured by scripts.

Moreover, the elimination of time and space constraints will provide contributions from a more varying population and include more young people. Collecting raw data from the platform prevents from a bias in the final report (e.g. the completeness of the information said is gathered, no interested writing, etc.). On the other hand, the utilization of a new online platform will imply that the citizens will need an adaptation and learning period. Furthermore, the retranscription of emotional aspects which are already lacking in public onsite meetings and will probably become more difficult. 


\section{CONCLUSIONS AND PERSPECTIVES}

The presented platform only requires a computer or mobile phone with an internet connection, we can therefore consider that the entry cost is relatively low. Compared to classic public engagement sessions, the main benefits of this concept are 1 . the removal of the temporal and spatial constraints of the participants (citizens and stakeholders) 2. the possibility to create an open, transparent debate 3 . the opportunity to reach larger parts of the population that otherwise cannot be included in a debate 4 . the advantage of using a 3D representation in terms of spatial cognition and spatial understanding as compared to the utilization of static 2D maps.

The 3D scene allows users to interact within a mutual cognition space and to share the same visual base between participants, moderators and stakeholders. Nevertheless, the use of a virtual platform as a mediator leads to a loss of some emotional information (e.g. body language) which can occur during discussions between stakeholders and citizens. This key factor can be collected during public on-site meetings using scribes. Another possibility to address this issue is to allow citizen to contribute via video posts (e.g. recorded via a webcam).

This project has not the ambition of replacing traditional public engagement meetings but to be combined with participatory citizen meetings and thereby to cumulate more data from other groups of the population that usually do not participate (e.g. due to time or space constraints).

At this stage of the project we have developed a working web app. We will soon test the prototype in a real-world scenario with citizen of a major Swiss city. This experience will allow us to refine the platform's interface and functionality as well as to respond to research questions such as: 1. Highlighting geovisualization codes for 3D map's representation. 2. How to shape of the platform (level of detail, functionality, data filtering, etc.) in accordance with the public (expert, non-expert) and the project (temporality, nature)? 3. How to gather effectively citizen tacit knowledge to meet authority expectations? 4. How to provide an automated effective and neutral feedback from the platform inputs?

\section{REFERENCES}

Bugs, G., Granell, C., Fonts, O., Huerta, J. \& Painho, M. 2010 An assessment of Public Participation GIS and Web 2.0 technologies in urban planning practice in Canela, Brazil. Cities 27, 172-181. 
Fechner, T. \& Kray, C. 2014 Georeferenced Open Data and Augmented Interactive Geo-Visualizations as Catalysts for Citizen Engagement. JeDEM - eJournal eDemocracy Open Gov. 6, 14-35.

Harvey, M. 2009 Drama, Talk, and Emotion. Sci. Technol. Hum. Values 34, 139-161.

Obama, Barack. 21/01/2009 Presidential speech, Remarks by the president in welcoming senior staff and cabinet secretaries to the white house. At the White House, Washington, D.C.

Sivarajah, U., Irani, Z. and Weerakkody, V. 2015 Evaluating the use and impact of Web 2.0 technologies in local government, Government Information Quarterly. Elsevier Inc., 32(4), pp. $473-487$. 